\title{
SOLVABILITY OF NONLINEAR DIRICHLET PROBLEM FOR A CLASS OF DEGENERATE ELLIPTIC EQUATIONS
}

ALBO CARLOS CAVALHEIRO

Received 7 August 2003

We prove an existence result for solution to a class of nonlinear degenerate elliptic equation associated with a class of partial differential operators of the form $L u(x)=$ $\sum_{i, j=1}^{n} D_{j}\left(a_{i j}(x) D_{i} u(x)\right)$, with $D_{j}=\partial / \partial x_{j}$, where $a_{i j}: \Omega \rightarrow \mathbb{R}$ are functions satisfying suitable hypotheses.

\section{Introduction}

In this paper, we prove the existence of solution in $D(A) \subseteq H_{0}(\Omega)$ for the following nonlinear Dirichlet problem:

$$
\begin{aligned}
-L u(x)+g(u(x)) \omega(x) & =f_{0}(x)-\sum_{j=1}^{n} D_{j} f_{j}(x) \text { on } \Omega, \\
u(x) & =0 \quad \text { on } \partial \Omega,
\end{aligned}
$$

where $L$ is an elliptic operator in divergence form

$$
L u(x)=\sum_{i, j=1}^{n} D_{j}\left(a_{i j}(x) D_{i} u(x)\right), \quad \text { with } D_{j}=\frac{\partial}{\partial x_{j}}
$$

and the coefficients $a_{i j}$ are measurable, real-valued functions whose coefficient matrix $\left(a_{i j}(x)\right)$ is symmetric and satisfies the degenerate ellipticity condition

$$
|\xi|^{2} \omega(x) \leq \sum_{i, j=1}^{n} a_{i j}(x) \xi_{i} \xi_{j} \leq|\xi|^{2} v(x)
$$

for all $\xi \in \mathbb{R}^{n}$ and almost every $x \in \Omega \subset \mathbb{R}^{n}$ a bounded open set with piecewise smooth boundary (i.e., $\partial \Omega \in C^{0,1}$ ), and $\omega$ and $v$ two weight functions (i.e., locally integrable nonnegative functions). 
The basic idea is to reduce (1.1) to an operator equation

$$
A u=T, \quad u \in D(A),
$$

where $D(A)=\left\{u \in H_{0}(\Omega): u(x) g(u(x)) \in L^{1}(\Omega, \omega)\right\}$, and apply the theorem below.

THeorem 1.1. Suppose that the following assumptions are satisfied.

(H1) Dual pairs. Let the dual pairs $\left\{X, X^{+}\right\}$and $\left\{Y, Y^{+}\right\}$be given, where $X, X^{+}, Y$, and $Y^{+}$are Banach spaces with corresponding bilinear forms $\langle\cdot, \cdot\rangle_{X}$ and $\langle\cdot, \cdot\rangle_{Y}$ and the continuous embeddings $Y \subseteq X$ and $X^{+} \subseteq Y^{+}$.

The dual pairs are compatible, that is,

$$
\langle T, u\rangle_{X}=\langle T, u\rangle_{Y}, \quad \forall T \in X^{+}, u \in Y .
$$

Moreover, the Banach spaces $X$ and $Y$ are separable and $X$ is reflexive.

(H2) Operator $A$. Let the operator $A: D(A) \subseteq X \rightarrow Y^{+}$be given, and let $K$ be a bounded closed convex set in $X$ containing the zero point as an interior point and $K \cap Y \subseteq D(A)$.

(H3) Local coerciveness. There exists a number $\alpha \geq 0$ such that $\langle A v, v\rangle_{Y} \geq \alpha$ for all $v \in Y \cap \partial K$, where $\partial K$ denotes the boundary of $K$ in the Banach space $X$.

(H4) Continuity. For each finite-dimensional subspace $Y_{0}$ of the Banach space $Y$, the mapping $u \mapsto\langle A u, v\rangle_{Y}$ is continuous on $K \cap Y_{0}$ for all $v \in Y_{0}$.

(H5) Generalized condition (M). Let $\left\{u_{n}\right\}$ be a sequence in $Y \cap K$ and let $T \in X^{+}$. Then, from

$$
\begin{gathered}
u_{n} \rightarrow u \quad \text { in } X \text { as } n \longrightarrow \infty, \\
\left\langle A u_{n}, v\right\rangle_{Y} \longrightarrow\langle T, v\rangle_{X} \quad \text { as } n \longrightarrow \infty, \forall v \in Y, \\
\varlimsup_{n \rightarrow \infty}\left\langle A u_{n}, u_{n}\right\rangle_{Y} \leq\langle T, u\rangle_{X},
\end{gathered}
$$

it follows that $A u=T$.

(H6) Quasiboundedness. Let $\left\{u_{n}\right\}$ be a sequence in $Y \cap K$. Then, from (1.6) and $\left\langle A u_{n}\right.$, $\left.u_{n}\right\rangle_{Y} \leq C\|u\|_{X}$ for all $n$, it follows that the sequence $\left\{A u_{n}\right\}$ is bounded in $Y^{+}$.

(H7) The operator $A$ is coercive, that is, $\langle A v, v\rangle_{Y} /\|v\|_{X} \rightarrow \infty$ as $\|v\|_{X} \rightarrow \infty, v \in Y$.

Then $X^{+} \subseteq R(A)$, that is, the equation $A u=T$ has a solution $u$ for each $T \in X^{+}$.

Proof. See [7, Theorem 27.B and Corollary 27.19].

We will apply this theorem to a sufficiently large ball $K$ in the Banach spaces $X=$ $H_{0}(\Omega), X^{+}=\left(H_{0}(\Omega)\right)^{*}$, and $Y^{+}=Y^{*}$.

We make the following basic assumption on the weights $\omega$ and $v$.

The weighted Sobolev inequality (WSI). Let $\Omega$ be an open bounded set in $\mathbb{R}^{n}$. There is an index $q=2 \sigma, \sigma>1$, such that for every ball $B$ and every $f \in \operatorname{Lip}_{0}(B)$ (i.e, $f \in \operatorname{Lip}(B)$ whose support is contained in the interior of $B$ ),

$$
\left(\frac{1}{v(B)} \int_{B}|f|^{q} v d x\right)^{1 / q} \leq C R_{B}\left(\frac{1}{\omega(B)} \int_{B}|\nabla f|^{2} \omega d x\right)^{1 / 2},
$$


with the constant $C$ independent of $f$ and $B, R_{B}$ the radius of $B$, and the symbol $\nabla$ indicating the gradient, $v(B)=\int_{B} v(x) d x$, and $\omega(B)=\int_{B} \omega(x) d x$.

Thus, we can write

$$
\left(\int_{B}|f|^{q} v d x\right)^{1 / q} \leq C_{B, \omega, v}\left(|\nabla f|^{2} \omega d x\right)^{1 / 2},
$$

where $C_{B, \omega, v}$ is called the Sobolev constant and

$$
C_{B, \omega, v}=\frac{C[v(B)]^{1 / q} R_{B}}{[\omega(B)]^{1 / 2}} .
$$

For instance, the WSI holds if $\omega$ and $v$ are as in [6, Chapter X, Theorem 4.8], or if $\omega$ and $v$ are as in [1, Theorem 1.5].

The following theorem will be proved in Section 3.

Theorem 1.2. Let $L$ be the operator (1.2) and satisfy (1.3). Suppose that the following assumptions are satisfied:

(i) $(v, \omega) \in A_{2}$;

(ii) the function $g: \mathbb{R} \rightarrow \mathbb{R}$ is continuous with $x g(x) \geq 0$ for all $x \in \mathbb{R}$;

(iii) $f_{0} / v \in L^{q^{\prime}}(\Omega, v)$ and $f_{j} / \omega \in L^{2}(\Omega, \omega), j=1,2, \ldots, n$ (where $q$ is as in WSI). Then problem (1.1) has solution $u \in D(A) \subseteq H_{0}(\Omega)$;

(iv) if the function $g: \mathbb{R} \rightarrow \mathbb{R}$ is monotone increasing, then the solution is unique.

Example 1.3. Consider the domain $\Omega=\left\{(x, y) \in \mathbb{R}^{2}:|x|<1\right.$ and $\left.|y|<1\right\}$. By Theorem 1.2 , the problem

$$
\begin{aligned}
-L u(x)+u(x, y) e^{u^{2}(x, y)}|x|^{1 / 2} & =1-\frac{\partial}{\partial x}\left(x^{2}|y|\right)-\frac{\partial}{\partial y}\left(y^{2}|x|\right) \quad \text { on } \Omega, \\
u(x, y) & =0 \quad \text { on } \partial \Omega,
\end{aligned}
$$

where

$$
L u(x)=\left[\frac{\partial}{\partial x}\left(|x|^{1 / 2} \frac{\partial u}{\partial x}\right)+\frac{\partial}{\partial y}\left(|x|^{-1 / 2} \frac{\partial u}{\partial y}\right)\right]
$$

has a unique solution $u \in D(A)=\left\{u \in H_{0}(\Omega): g(u(x, y)) u(x, y) \in L^{1}(\Omega, \omega)\right\}$, where $g(t)$ $=t e^{t^{2}}, \omega(x, y)=|x|^{1 / 2}, v(x, y)=|x|^{-1 / 2}, f_{0}(x, y)=1, f_{1}(x, y)=x^{2}|y|$, and $f_{2}(x, y)=$ $y^{2}|x|$.

\section{Definitions and basic results}

Let $\omega$ be a locally integrable nonnegative function in $\mathbb{R}^{n}$ and assume that $0<\omega<\infty$ almost everywhere. We say that $\omega$ belongs to the Muckenhoupt class $A_{p}, 1<p<\infty$, or that $\omega$ is an $A_{p}$-weight if there is a constant $C_{1}=C(p, \omega)$ such that

$$
\left(\frac{1}{|B|} \int_{B} \omega(x) d x\right)\left(\frac{1}{|B|} \int_{B} \omega^{1 /(1-p)}(x) d x\right)^{p-1} \leq C_{1},
$$


for all balls $B \subset \mathbb{R}^{n}$, where $|\cdot|$ denotes the $n$-dimensional Lebesgue measure in $\mathbb{R}^{n}$. If $1<q \leq p$, then $A_{q} \subset A_{p}$ (see $[4,5]$ for more information about $A_{p}$-weights). The weight $\omega$ satisfies the doubling condition if $\omega(2 B) \leq C \omega(B)$, for all balls $B \subset \mathbb{R}^{n}$, where $\omega(B)=$ $\int_{B} \omega(x) d x$ and $2 B$ denotes the ball with the same center as $B$ which is twice as large. If $\omega \in A_{p}$, then $\omega$ is doubling (see [5, Corollary 15.7]).

We say that the pair of weights $(\nu, \omega)$ satisfies the condition $A_{p}(1<p<\infty$ and $(\nu, \omega) \in$ $A_{p}$ ) if and only if there is a constant $C_{2}$ such that

$$
\left(\frac{1}{|B|} \int_{B} v(x) d x\right)\left(\frac{1}{|B|} \int_{B} \omega^{1 /(1-p)}(x) d x\right)^{p-1} \leq C_{2}
$$

for every ball $B \subset \mathbb{R}^{n}$.

Remark 2.1. If $(v, \omega) \in A_{p}$ and $\omega \leq v$, then $\omega \in A_{p}$ and $v \in A_{p}$.

Given a measurable subset $\Omega$ of $\mathbb{R}^{n}$, we will denote by $L^{p}(\Omega, \omega), 1 \leq p<\infty$, the Banach space of all measurable functions $f$ defined on $\Omega$ for which

$$
\|f\|_{L^{p}(\Omega, \omega)}=\left(\int_{\Omega}|f(x)|^{p} \omega(x) d x\right)^{1 / p}<\infty .
$$

We will denote by $W^{k, p}(\Omega, \omega)$, the weighted Sobolev spaces, the set of all functions $u \in L^{p}(\Omega, \omega)$ such that the weak derivatives $D^{\alpha} u \in L^{p}(\Omega, \omega), 1 \leq|\alpha| \leq k$. The norm in the space $W^{k, p}(\Omega, \omega)$ is defined by

$$
\|u\|_{W^{k, p}(\Omega, \omega)}=\left(\int_{\Omega}|u(x)|^{p} \omega(x) d x+\sum_{1 \leq|\alpha| \leq k} \int_{\Omega}\left|D^{\alpha} u(x)\right|^{p} \omega(x) d x\right)^{1 / p} .
$$

If $\omega \in A_{p}$, then $W^{k, p}(\Omega, \omega)$ is the closure of $C^{\infty}(\bar{\Omega})$ with respect to the norm (2.4) (see [2, Proposition 3.5]). The space $W_{0}^{k, p}(\Omega, \omega)$ is the closure of $C_{0}^{\infty}(\Omega)$ with respect to the norm

$$
\|u\|_{W_{0}^{k, p}(\Omega, \omega)}=\left(\sum_{1 \leq|\alpha| \leq k} \int_{\Omega}\left|D^{\alpha} u(x)\right|^{p} \omega(x) d x\right)^{1 / p}
$$

When $k=1$ and $p=2$, the spaces $W^{1,2}(\Omega, \omega)$ and $W_{0}^{1,2}(\Omega, \omega)$ are Hilbert spaces. We will denote by $H_{0}(\Omega)$ the closure of $C_{0}^{\infty}(\bar{\Omega})$ with respect to the norm

$$
\|u\|_{H_{0}(\Omega)}=\left(\int_{\Omega}\langle\mathscr{A}(x) \nabla u(x), \nabla u(x)\rangle d x\right)^{1 / 2},
$$

where $\mathscr{A}(x)=\left[a_{i j}(x)\right]$ (the coefficient matrix) and the symbol $\nabla$ indicates the gradient. 
Remark 2.2. Using the condition (1.3), we have

$$
\begin{gathered}
\|u\|_{W_{0}^{1,2}(\Omega, \omega)} \leq\|u\|_{H_{0}(\Omega)} \leq\|u\|_{W_{0}^{1,2}(\Omega, v)}, \\
W_{0}^{1,2}(\Omega, v) \subset H_{0}(\Omega) \subset W_{0}^{1,2}(\Omega, \omega) .
\end{gathered}
$$

Lemma 2.3. If $\omega \in A_{2}$, then $W_{0}^{1,2}(\Omega, \omega) \hookrightarrow L^{2}(\Omega, \omega)$ is compact and

$$
\|u\|_{L^{2}(\Omega, \omega)} \leq C_{3}\|u\|_{W_{0}^{1,2}(\Omega, \omega)}
$$

Proof. The proof follows the lines of [3, Theorem 4.6].

We introduce the following definition of (weak) solutions for problem (1.1).

Definition 2.4. A function $u \in D(A) \subseteq H_{0}(\Omega)$ is (weak) solution to the problem (1.1) if

$$
\begin{gathered}
\int_{\Omega} a_{i j}(x) D_{i} u(x) D_{j} \varphi(x) d x+\int_{\Omega} g(u(x)) \varphi(x) \omega(x) d x \\
=\int_{\Omega} f_{0}(x) \varphi(x) d x+\int_{\Omega} f_{j}(x) D_{j} \varphi(x) d x
\end{gathered}
$$

for all $\varphi \in Y=H_{0}(\Omega) \cap W^{k, p}(\Omega, v)$, where $p>4, k>n / 2$, and $\|\varphi\|_{Y}=\|\varphi\|_{W^{k, p}(\Omega, v)}$, with $D(A)=\left\{u \in H_{0}(\Omega): g(u(x)) u(x) \in L^{1}(\Omega, \omega)\right\}$.

Remark 2.5. Using that $p>4$, we have that $v \in A_{2} \subset A_{p / 2}$ and

$$
\|\cdot\|_{L^{2}(\Omega)} \leq\left[v^{1 /(1-p / 2)}(\Omega)\right]^{(p-2) / 2 p}\|\cdot\|_{L^{p}(\Omega, v)} .
$$

Thus, $W^{k, p}(\Omega, v) \subset W^{k, 2}(\Omega) \subset C(\bar{\Omega})$ (by the Sobolev embedding theorem).

Therefore $\|\cdot\|_{C(\bar{\Omega})} \leq C\|\cdot\|_{Y}$ and the embedding $Y \subset C(\bar{\Omega})$ is continuous.

\section{Proof of Theorem 1.2}

(I) Existence. For $u \in D(A)$ and $\varphi \in Y$, we define

$$
\begin{aligned}
B_{1}(u, \varphi) & =\int_{\Omega} a_{i j}(x) D_{i} u(x) D_{j} \varphi(x) d x, \\
B_{2}(u, \varphi) & =\int_{\Omega} g(u(x)) \varphi(x) \omega(x) d x \\
T(\varphi) & =\int_{\Omega} f_{0}(x) \varphi(x) d x+\sum_{j=1}^{n} \int_{\Omega} f_{j}(x) D_{j} \varphi(x) d x .
\end{aligned}
$$

Then $u \in D(A) \subseteq H_{0}(\Omega)$ is solution to problem (1.1) if

$$
B_{1}(u, \varphi)+B_{2}(u, \varphi)=T(\varphi), \quad \forall \varphi \in Y
$$


210 Solvability for a class of nonlinear Dirichlet problem

Step $1\left(T \in\left(H_{0}(\Omega)\right)^{*}\right)$. In fact, using hypothesis (iii), Lemma 2.3, the Hölder inequality, the WSI, and (2.7), we obtain

$$
\begin{aligned}
|T(\varphi)| & \leq \int_{\Omega}\left|f_{0}\right||\varphi| d x+\sum_{j=1}^{n} \int_{\Omega}\left|f_{j}\right|\left|D_{j} \varphi\right| d x \\
& =\int_{\Omega}\left(\frac{\left|f_{0}\right|}{v}\right) v^{1 / q^{\prime}}|\varphi| v^{1 / q} d x+\sum_{j=1}^{n} \int_{\Omega}\left(\frac{\left|f_{j}\right|}{\omega}\right) \omega^{1 / 2}\left|D_{j} \varphi\right| \omega^{1 / 2} d x \\
& \leq\left\|\frac{f_{0}}{v}\right\|_{L^{q^{\prime}}(\Omega, v)}\|\varphi\|_{L^{q}(\Omega, v)}+\sum_{j=1}^{n}\left\|\frac{f_{j}}{\omega}\right\|_{L^{2}(\Omega, \omega)}\left\|D_{j} \varphi\right\|_{L^{2}(\Omega, \omega)} \\
& \leq C_{B, \omega, v}\left\|\frac{f_{0}}{v}\right\|_{L^{q^{\prime}}(\Omega, v)}\|\nabla \varphi\|_{L^{2}(\Omega, \omega)}+\sum_{j=1}^{n}\left\|\frac{f_{j}}{\omega}\right\|_{L^{2}(\Omega, \omega)}\|\nabla \varphi\|_{L^{2}(\Omega, \omega)} \\
& \leq C\left(\left\|\frac{f_{0}}{v}\right\|_{L^{q^{\prime}}(\Omega, v)}+\sum_{j=1}^{n}\left\|\frac{f_{j}}{\omega}\right\|_{L^{2}(\Omega, \omega)}\right)\|\varphi\|_{H_{0}(\Omega), \quad \forall \varphi \in H_{0}(\Omega) .}
\end{aligned}
$$

Step 2. By condition (1.3) and the hypothesis that the matrix $\mathscr{A}$ is symmetric, we obtain

$$
\begin{aligned}
\left|B_{1}(u, \varphi)\right| & \leq \int_{\Omega} \operatorname{big}|\langle\mathscr{A} \nabla u, \nabla \varphi\rangle| d x \\
& \leq \int_{\Omega}\langle\mathscr{A} \nabla u, \nabla u\rangle^{1 / 2}\langle\mathscr{A} \nabla \varphi, \nabla \varphi\rangle^{1 / 2} d x \\
& \leq\|u\|_{H_{0}(\Omega)}\|\varphi\|_{H_{0}(\Omega)} \\
& \leq\|u\|_{H_{0}(\Omega)}\|\varphi\|_{W_{0}^{1,2}(\Omega, v)} \\
& \leq\|u\|_{H_{0}(\Omega)}\|\varphi\|_{Y},
\end{aligned}
$$

for all $u \in H_{0}(\Omega), \varphi \in Y$.

Hence there exists exactly one linear continuous operator

$$
A_{1}: H_{0}(\Omega) \longrightarrow Y^{*}
$$

with

$$
\left\langle A_{1} u, \varphi\right\rangle_{Y}=B_{1}(u, \varphi), \quad \forall u \in H_{0}(\Omega), \varphi \in Y
$$

Step 3. Note that $|g(x)| \leq x g(x)+C_{4}$, for all $x \in \mathbb{R}$. Therefore, if $u \in D(A)$, we have that $g(u(x)) \in L^{1}(\Omega, \omega)$. By using hypothesis (ii), Lemma 2.3, and Remark 2.5, we obtain for $u \in D(A)$ fixed

$$
\begin{aligned}
\left|B_{2}(u, \varphi)\right| & \leq \int_{\Omega}|g(u(x))||\varphi(x)| \omega(x) d x \\
& \leq\|\varphi\|_{C(\bar{\Omega})} \int_{\Omega}|g(u(x))| \omega(x) d x \\
& \leq C\|\varphi\|_{Y} .
\end{aligned}
$$


Thus, there exists a unique operator

$$
A_{2}: D(A) \subseteq H_{0}(\Omega) \longrightarrow Y^{*},
$$

with

$$
\left\langle A_{2} u, \varphi\right\rangle_{Y}=B_{2}(u, \varphi), \quad \forall u \in D(A), \varphi \in Y
$$

Step 4. We define the operator

$$
A: D(A) \subseteq H_{0}(\Omega) \longrightarrow Y^{*}, \quad A=A_{1}+A_{2} .
$$

We have

$$
\langle A u, \varphi\rangle_{Y}=\left\langle A_{1} u, \varphi\right\rangle_{Y}+\left\langle A_{2} u, \varphi\right\rangle_{Y}=B_{1}(u, \varphi)+B_{2}(u, \varphi) .
$$

Thus, $u \in D(A)$ is a solution to problem (1.1) if

$$
\langle A u, \varphi\rangle_{Y}=T(\varphi), \quad \forall \varphi \in Y .
$$

Then, the problem (1.1) corresponds to the operator equation (1.4).

Step 5. Global coerciveness of operator A. Using the condition (1.3) and hypothesis (ii), we obtain

$$
\begin{aligned}
\langle A \varphi, \varphi\rangle_{Y} & =B_{1}(\varphi, \varphi)+B_{2}(\varphi, \varphi) \\
& =\int_{\Omega} a_{i j}(x) D_{i} \varphi(x) D_{j} \varphi(x) d x+\int_{\Omega} g(\varphi(x)) \varphi(x) \omega(x) d x \\
& \geq \int_{\Omega}\langle A \mathcal{} \nabla \varphi, \nabla \varphi\rangle d x \\
& =\|\varphi\|_{H_{0}(\Omega)}^{2} .
\end{aligned}
$$

Thus

$$
\lim _{\|\varphi\|_{H_{0}(\Omega)} \rightarrow \infty} \frac{\langle A \varphi, \varphi\rangle_{Y}}{\|\varphi\|_{H_{0}(\Omega)}}=+\infty .
$$

Step 6. Generalized condition $(M)$. Let $T \in\left(H_{0}(\Omega)\right)^{*}$ and let $\left\{u_{n}\right\}$ be a sequence in $Y$ with

$$
\begin{gathered}
u_{n} \rightarrow u \quad \text { in } H_{0}(\Omega), \\
\left\langle A u_{n}, \varphi\right\rangle_{Y} \longrightarrow T(\varphi) \quad \text { as } n \longrightarrow \infty, \forall \varphi \in Y, \\
\varlimsup_{n \rightarrow \infty}\left\langle A u_{n}, u_{n}\right\rangle \leq T(u) .
\end{gathered}
$$

We want to show that this implies that $A u=T$.

Using that the operator $A_{1}$ is linear and continuous, we obtain

$$
\left\langle A_{1} u_{n}, \varphi\right\rangle_{Y} \longrightarrow\left\langle A_{1} u, \varphi\right\rangle_{Y}, \quad \forall \varphi \in Y
$$


212 Solvability for a class of nonlinear Dirichlet problem

Because of (3.16), it is sufficient to prove that $u \in D(A)$ and

$$
\left\langle A_{2} u_{n}, \varphi\right\rangle_{Y} \longrightarrow\left\langle A_{2} u, \varphi\right\rangle_{Y}, \quad \forall \varphi \in Y
$$

Therefore, it is sufficient to show that

$$
\int_{\Omega}\left[g\left(u_{n}(x)\right)-g(u(x))\right] \varphi(x) \omega(x) d x \longrightarrow 0 \quad \text { as } n \longrightarrow \infty .
$$

Using the same argument in Step 3, we obtain

$$
\begin{aligned}
& \left|\int_{\Omega}\left(g\left(u_{n}(x)\right)-g(u(x))\right) \varphi(x) \omega(x) d x\right| \\
& \quad \leq \int_{\Omega}\left|g\left(u_{n}(x)\right)-g(u(x))\right||\varphi(x)| \omega(x) d x \\
& \quad \leq\|\varphi\|_{C(\bar{\Omega})} \int_{\Omega}\left|g\left(u_{n}(x)\right)-g(u(x))\right| \omega(x) d x \\
& \quad \leq C\|\varphi\|_{Y} \int_{\Omega}\left|g\left(u_{n}(x)\right)-g(u(x))\right| \omega(x) d x .
\end{aligned}
$$

Therefore, it is sufficient to show that

$$
g\left(u_{n}(x)\right) \longrightarrow g(u(x)) \quad \text { in } L^{1}(\Omega, \omega) .
$$

Note that it is sufficient to prove (3.22) for a subsequence of $\left\{u_{n}\right\}$.

If $(v, \omega) \in A_{2}$ and $\omega \leq v$, then $\omega \in A_{2}$ (see Remark 2.1). By Lemma 2.3,

$$
W_{0}^{1,2}(\Omega, \omega) \hookrightarrow L^{2}(\Omega, \omega)
$$

is compact and $\|u\|_{L_{2}(\Omega, \omega)} \leq C_{2}\|u\|_{W_{0}^{1,2}(\Omega, \omega)}$. Using (2.7), we also have that

$$
H_{0}(\Omega) \hookrightarrow L^{2}(\Omega, \omega)
$$

is compact. This implies $u_{n} \rightarrow u$ in $L^{2}(\Omega, \omega)$. Using again that $\omega \in A_{2}$, we have $u_{n} \rightarrow u$ in $L^{1}(\Omega)$. Thus, there exists a subsequence, again denoted by $\left\{u_{n}\right\}$, such that $u_{n}(x) \rightarrow u(x)$ for almost all $x \in \Omega$. The continuity of $g$ implies that $g\left(u_{n}(x)\right) \rightarrow g(u(x))$ for almost all $x \in \Omega$. Moreover, since $u_{n} \rightarrow u$ in $H_{0}(\Omega)$, it follows that

$$
\sup \left\|u_{n}\right\|_{H_{0}(\Omega)} \leq C, \quad \text { independent of } n \text {. }
$$

Hence, using (1.2), we obtain

$$
\left\langle A_{1} u_{n}, u_{n}\right\rangle_{Y} \leq \Lambda\left\|u_{n}\right\|_{H_{0}(\Omega)}^{2} \leq \Lambda C^{2}, \quad \text { with } C \text { independent of } n \text {. }
$$

Therefore, using (3.16), we obtain

$$
\varlimsup_{n \rightarrow \infty}\left\langle A_{2} u_{n}, u_{n}\right\rangle_{Y}=\varlimsup_{n \rightarrow \infty} \int_{\Omega} g\left(u_{n}(x)\right) u_{n}(x) \omega(x) d x \leq C,
$$

with $C$ independent of $n$. 
The continuity of $g$ implies that $g\left(u_{n}(x)\right) u_{n}(x) \omega(x) \rightarrow g(u(x)) u(x) \omega(x)$ for almost all $x \in \Omega$. Therefore, by Fatou lemma, we have

$$
\int_{\Omega} g(u(x)) u(x) \omega(x) d x<\infty
$$

that is, $u \in D(A)$.

Now we want to show that $g\left(u_{n}(x)\right) \rightarrow g(u(x))$ in $L^{1}(\Omega, \omega)$.

Let $a>0$ be fixed. For each $x \in \Omega$, we have either

$$
\left|u_{n}(x)\right| \leq a \quad \text { or } \quad\left|g\left(u_{n}(x)\right)\right| \leq a^{-1} g\left(u_{n}(x)\right) u_{n}(x)
$$

(if $x \neq 0$, we can write $g(x)=x^{-1}[g(x) x]$ ). We get $|g(x)| \leq c(a)$ if $|x| \leq a$ (because $g$ is continuous).

Let $X$ be a measurable subset of $\Omega$. Then

$$
\begin{aligned}
\int_{X}\left|g\left(u_{n}(x)\right)\right| \omega(x) d x= & \int_{X \cap\left\{x:\left|u_{n}(x)\right| \leq a\right\}}\left|g\left(u_{n}(x)\right)\right| \omega(x) d x \\
& +\int_{X \cap\left\{x:\left|u_{n}(x)\right|>a\right\}}\left|g\left(u_{n}(x)\right)\right| \omega(x) d x \\
\leq & c(a) \omega(X)+a^{-1} \int_{X} g\left(u_{n}(x)\right) u_{n}(x) \omega(x) d x \\
\leq & c(a) \omega(X)+a^{-1} C \quad(\text { by }(3.27)) .
\end{aligned}
$$

Hence, for all $\varepsilon>0$, we have

$$
\int_{X}\left|g\left(u_{n}(x)\right)\right| \omega(x) d x \leq \frac{\varepsilon}{2}
$$

if $a$ is sufficiently large and $\omega(X)$ is sufficiently small. Therefore, for all $\varepsilon>0$, there exists $\delta=\delta(\varepsilon)$ such that

$$
\begin{aligned}
& \int_{X}\left|g\left(u_{n}(x)\right)-g(u(x))\right| \omega(x) d x \\
& \quad \leq \int_{X}\left|g\left(u_{n}(x)\right)\right| \omega(x) d x+\int_{X}|g(u(x))| \omega(x) d x \leq \varepsilon,
\end{aligned}
$$

with $\omega(X)<\delta$. Thus, the Vitali convergence theorem tells us that (3.22) holds.

Step 7. Quasiboundedness of the operator $A$. Let $\left\{u_{n}\right\}$ be a sequence in $Y$ with $u_{n} \rightarrow u$ in $H_{0}(\Omega)$ and suppose that

$$
\left\langle A u_{n}, u_{n}\right\rangle_{Y} \leq C\left\|u_{n}\right\|_{H_{0}(\Omega)}, \quad \forall n
$$

We want to show that the sequence $\left\{A u_{n}\right\}$ is bounded in $Y^{*}$. In fact, the boundedness of $\left\{u_{n}\right\}$ in $H_{0}(\Omega)$ implies that

$$
\varlimsup_{n \rightarrow \infty}\left\langle A u_{n}, u_{n}\right\rangle_{Y} \leq C
$$




\section{Solvability for a class of nonlinear Dirichlet problem}

Suppose by contradiction that the sequence $\left\{A u_{n}\right\}$ is unbounded in $Y^{*}$. Then there exists a subsequence, again denoted by $\left\{u_{n}\right\}$, such that

$$
\left\|A u_{n}\right\|_{Y^{*}} \longrightarrow \infty \text { as } n \longrightarrow \infty \text {. }
$$

By the same arguments as in Step 6, we obtain that

$$
\left\langle A u_{n}, \varphi\right\rangle_{Y} \longrightarrow\langle A u, \varphi\rangle_{Y} \quad \text { as } n \longrightarrow \infty, \forall \varphi \in Y .
$$

The uniform boundedness principle tells us that the sequence $\left\{A u_{n}\right\}$ is bounded (which is a contradiction with (3.35)).

Therefore, by Theorem 1.1, the equation $A u=T$, with $T \in\left(H_{0}(\Omega)\right)^{*}$, has a solution $u \in D(A) \subseteq H_{0}(\Omega)$, and it is the solution for problem (1.1).

(II) Uniqueness. If the function $g: \mathbb{R} \rightarrow \mathbb{R}$ is monotone increasing, we have that $(g(a)-$ $g(b))(a-b) \geq 0$, for all $a, b \in \mathbb{R}$. Then

$$
\begin{aligned}
\langle A u-A v, u-v\rangle_{Y}= & \int_{\Omega}\langle\mathscr{A} \nabla(u-v), \nabla(u-v)\rangle d x \\
& +\int_{\Omega}(g(u(x))-g(v(x)))(u(x)-v(x)) \omega(x) d x \\
\geq & \int_{\Omega}\langle\mathscr{A} \nabla(u-v), \nabla(u-v)\rangle d x=\|u-v\|_{H_{0}(\Omega)}^{2},
\end{aligned}
$$

for all $u, v \in D(A)$.

Therefore, if $u, v \in D(A)$ and $A u=A v=T$, we obtain that $u=v$.

\section{References}

[1] S. Chanillo and R. L. Wheeden, Weighted Poincaré and Sobolev inequalities and estimates for weighted Peano maximal functions, Amer. J. Math. 107 (1985), no. 5, 1191-1226.

[2] V. Chiadò Piat and F. Serra Cassano, Relaxation of degenerate variational integrals, Nonlinear Anal. 22 (1994), no. 4, 409-424.

[3] B. Franchi and R. Serapioni, Pointwise estimates for a class of strongly degenerate elliptic operators: a geometrical approach, Ann. Scuola Norm. Sup. Pisa Cl. Sci. (4) 14 (1987), no. 4, $527-568$.

[4] J. García-Cuerva and J. L. Rubio de Francia, Weighted Norm Inequalities and Related Topics, North-Holland Mathematics Studies, vol. 116, North-Holland Publishing, Amsterdam, 1985.

[5] J. Heinonen, T. Kilpeläinen, and O. Martio, Nonlinear Potential Theory of Degenerate Elliptic Equations, Oxford Mathematical Monographs, The Clarendon Press, Oxford University Press, New York, 1993.

[6] A. Torchinsky, Real-Variable Methods in Harmonic Analysis, Pure and Applied Mathematics, vol. 123, Academic Press, Florida, 1986.

[7] E. Zeidler, Nonlinear Functional Analysis and Its Applications. II/B, Springer-Verlag, New York, 1990.

Albo Carlos Cavalheiro: Departamento de Matemática, Universidade Estadual de Londrina, Londrina, PR 86051-990, Brazil

E-mail address: albo@uel.br 


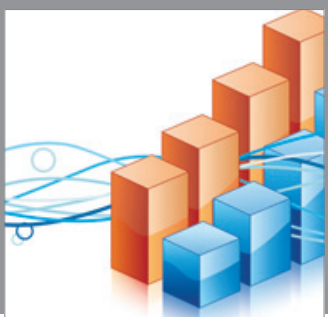

Advances in

Operations Research

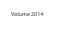

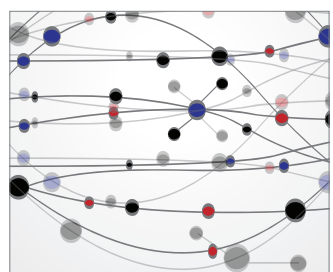

\section{The Scientific} World Journal
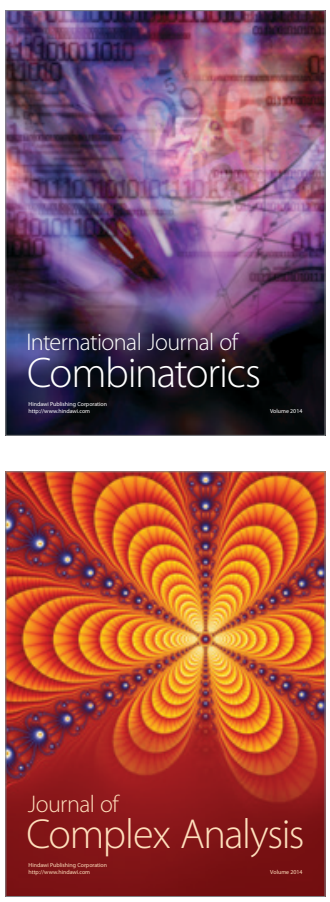

International Journal of

Mathematics and

Mathematical

Sciences
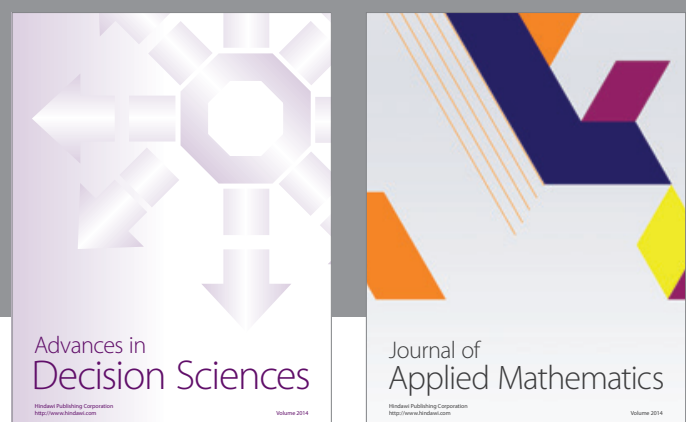

Journal of

Applied Mathematics
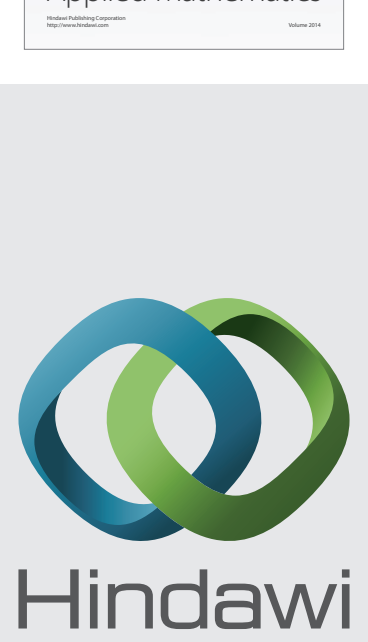

Submit your manuscripts at http://www.hindawi.com
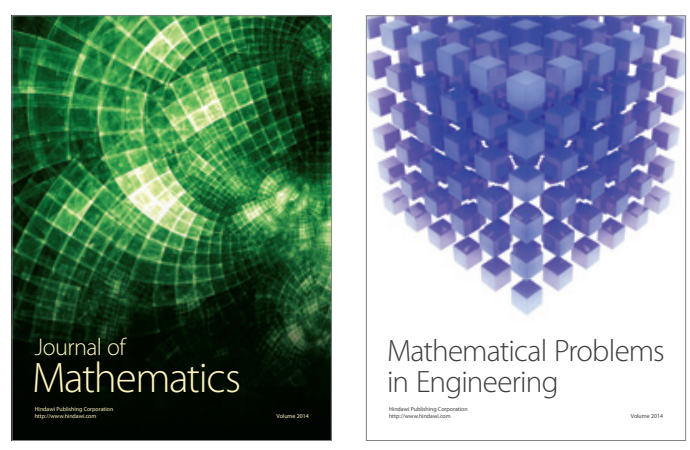

Mathematical Problems in Engineering
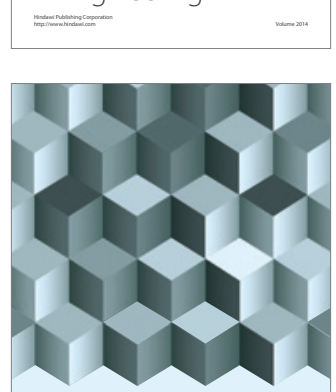

Journal of

Function Spaces
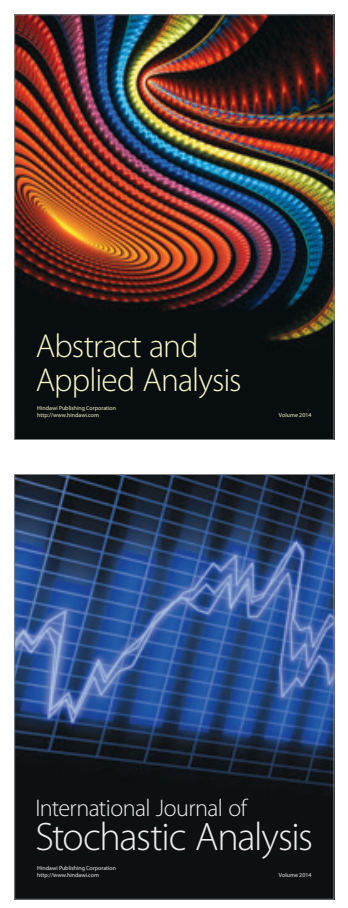

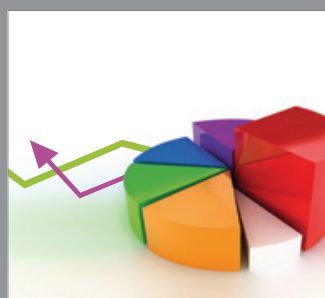

ournal of

Probability and Statistics

Promensencen
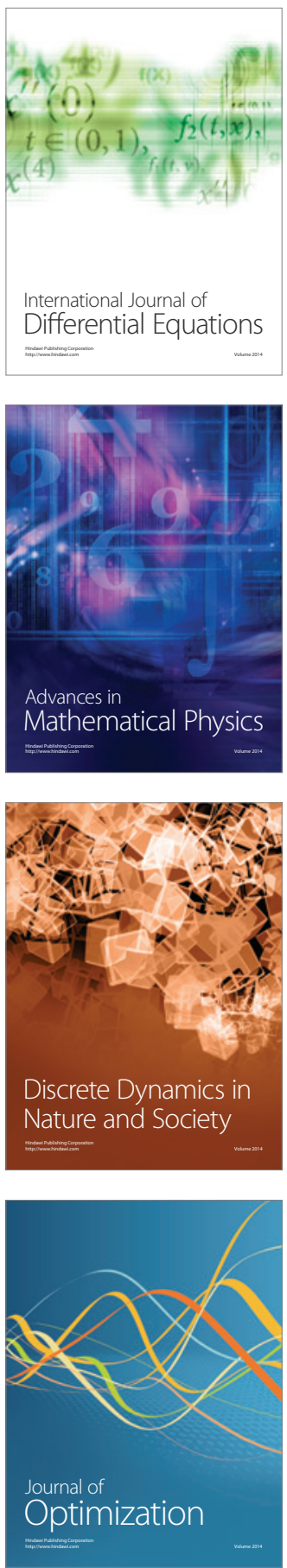